\title{
Establishing a multiple structures AHP evaluation by extending Hierarchies Consistency Analysis
}

\author{
Y-Y Guh ${ }^{1}$, H-M Wee ${ }^{2}$ and R-W Po ${ }^{3}$ \\ ${ }^{1}$ Graduate School of Business Administration Chung Yuan Christian University, \\ Chung li 320, Taiwan, R.O.C. \\ ${ }^{2}$ Department of Industrial Engineering, Chung Yuan Christian University \\ Chung li 320, Taiwan, R.O.C. \\ ${ }^{3}$ Graduate School of Technology Management, Chung Hua University \\ Hsinchu 300, Taiwan, R.O.C
}

\begin{abstract}
The purpose of this paper is to extend the theory of Hierarchies Consistency Analysis (HCA) to establish a multiple structures evaluation model for the AHP. Traditionally, the AHP process is based on a single evaluation hierarchy, according to statistics and group decision theories, a more objective evaluation will be obtained if we can compromise several estimates. We will construct several different structure schemata for a problem to be analyzed by the AHP, and determine the weight of criteria for all levels of each structure. Finally, we use the weight consistent property and compromise process of the HCA to integrate these AHP models to obtain more objective evaluation result that is as free as possible from structure bias.
\end{abstract}

The multiple structures of AHP model can be regarded as a generalized AHP model; hence the AHP model based on only one hierarchy structure is a special instance of the proposed method.

Keywords : AHP 、 Hierarchical Weighting 、 Group Decision 、 Evaluation - Multiple Attribute

\section{Introduction}

AHP is a multiple-criterion decision making methodology that was developed by Saaty in $1971[1,2,3]$. For years, the practical nature of the method, suitable for solving complicated and elusive evaluation problems, has been applied in highly diverse fields such as resource allocation, performance measurement, alternative selection, project evaluation and public policy analysis. The AHP process begins with setting up an evaluation hierarchy structure for the problem based upon interrelated criteria drawn from the problem itself Next, a matrix of pair-wise comparisons of the interrelated criteria for each clusters of the hierarchy is made by the Decision Maker (DM). Finally, the relative weight of criteria is obtained by utilizing the eigenvalue method. With the acquisition of the largest eigenvalue from the comparison matrix, a consistency index is constructed to measure the degree of rationality of the DM in making pair-wise comparisons.

The HCA method, developed by Guh in 1996 [4, 5, 6], considers the analysis of an evaluation problem. The DM must, therefore, create various evaluation hierarchy structures based on different viewpoints. According to the consistent weight property, the HCA methodology integrates these different hierarchy structures to obtain compromised weight for all levels and for all hierarchy structures. Taking the evaluation of faculty performance as an example will show the problem more succinctly. If the evaluation criteria of lowest level is divided into the quality of teaching $\left(\mathrm{x}_{11}\right)$, quantity of teaching $\left(\mathrm{x}_{12}\right)$, quality of research $\left(\mathrm{x}_{21}\right)$, quantity of research $\left(\mathrm{x}_{22}\right)$, quality of $\operatorname{service}\left(\mathrm{x}_{31}\right)$ and quantity of service $\left(\mathrm{x}_{32}\right)$, then each of the four instances may be appropriate as an evaluation structure. (See Figure 1).
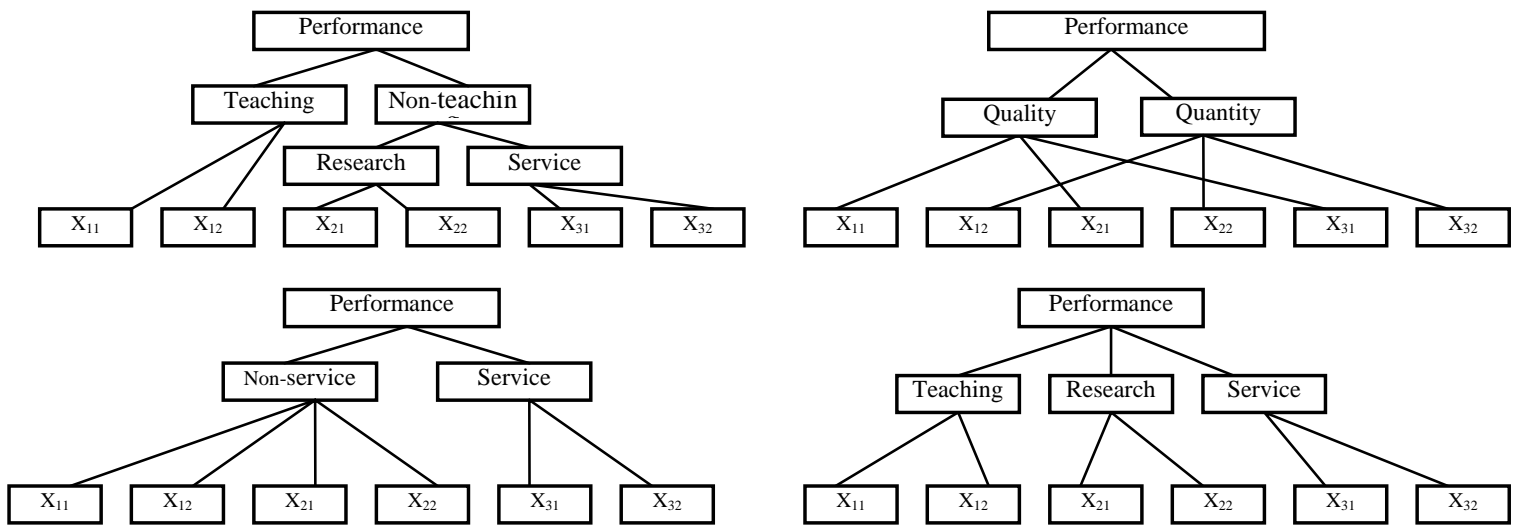

Figure 1 Multiple structures model of HCA 
A multiple structures model for the faculty performance evaluation problem is developed here. Through the AHP process and the use of HCA theory, we integrate these AHP models to obtain a consistent and compromised weight for all levels of hierarchy structures. So that it can be obtained a more objective evaluation result that is as free as possible from structure bias.

\section{HCA Concept}

HCA is required to first define the lowest level of criteria, and accordingly to construct different evaluation hierarchy structures depending on different viewpoints. Let us consider the problem illustrated in Figure 2

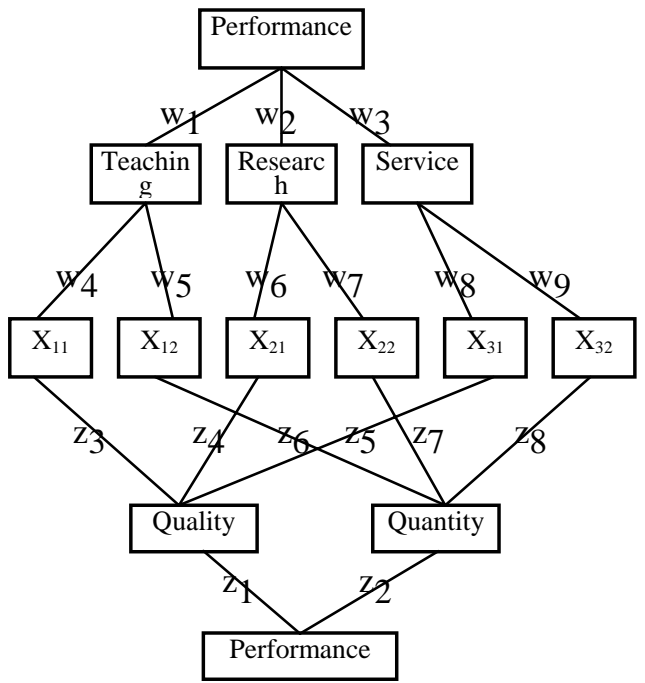

Figure 2 the illustration of HCA Concept

These two structures, with the same lowest level evaluation criteria, which has different aggregated path as associated with different hierarchy structures. Within the weights of criteria in these two structures, it can be deduced the following transformation relationship:

$$
\begin{aligned}
& \mathrm{z}_{1}=\mathrm{w}_{1} \mathrm{w}_{4}+\mathrm{w}_{2} \mathrm{w}_{6}+\mathrm{w}_{3} \mathrm{w}_{8} \\
& \mathrm{z}_{2}=\mathrm{w}_{1} \mathrm{w}_{5}+\mathrm{w}_{2} \mathrm{w}_{7}+\mathrm{w}_{3} \mathrm{w}_{9} \\
& \mathrm{z}_{3}=\mathrm{w}_{1} \mathrm{w}_{4} /\left(\mathrm{w}_{1} \mathrm{w}_{4}+\mathrm{w}_{2} \mathrm{w}_{6}+\mathrm{w}_{3} \mathrm{w}_{8}\right) \\
& \mathrm{z}_{4}=\mathrm{w}_{2} \mathrm{w}_{6} /\left(\mathrm{w}_{1} \mathrm{w}_{4}+\mathrm{w}_{2} \mathrm{w}_{6}+\mathrm{w}_{3} \mathrm{w}_{8}\right) \\
& \mathrm{z}_{5}=\mathrm{w}_{3} \mathrm{w}_{8} /\left(\mathrm{w}_{1} \mathrm{w}_{4}+\mathrm{w}_{2} \mathrm{w}_{6}+\mathrm{w}_{3} \mathrm{w}_{8}\right) \\
& \mathrm{z}_{6}=\mathrm{w}_{1} \mathrm{w}_{5} /\left(\mathrm{w}_{1} \mathrm{w}_{5}+\mathrm{w}_{2} \mathrm{w}_{7}+\mathrm{w}_{3} \mathrm{w}_{9}\right) \\
& \mathrm{z}_{7}=\mathrm{w}_{2} \mathrm{w}_{7} /\left(\mathrm{w}_{1} \mathrm{w}_{5}+\mathrm{w}_{2} \mathrm{w}_{7}+\mathrm{w}_{3} \mathrm{w}_{9}\right) \\
& \mathrm{z}_{8}=\mathrm{w}_{3} \mathrm{w}_{9} /\left(\mathrm{w}_{1} \mathrm{w}_{5}+\mathrm{w}_{2} \mathrm{w}_{7}+\mathrm{w}_{3} \mathrm{w}_{9}\right)
\end{aligned}
$$

Therefore, if the weight of criteria in these two structures satisfies the above relationship, then we say that both structures possess the consistent weights, and the same aggregated evaluation index should be obtained for the same evaluated object. Based on the consistent weight property, a consistency index is developed to inspect the degree of consistency of DM assigned weight to each criterion for all hierarchy structures, and the measure can be used to judge and to improve the process of weight assignment.

\section{Multiple Structures Model}

Assume that a large-scale problem contains m0 components of the lowest level criteria, there then exist Type 1, Type 2,...,Type s evaluation hierarchy structures, respectively constructed by DM from different viewpoints(see the upper hierarchies in Figure 3). ${ }_{i} W_{j}^{0}, \mathrm{i}=1$ to $\mathrm{s}, \mathrm{j}=1$ to mi, denotes the prior weights that are assigned by DM for each hierarchy structure.

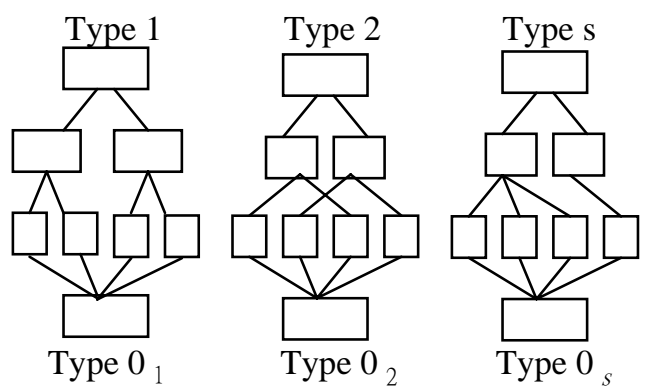

Figure 3. Multiple structures model of HCA

Initially we create one-level evaluation structures for the Type 1, Type 2,...,Type s hierarchy structures and number them as Type $0_{1}$, Type $0_{2}, \ldots$,Type $0_{s}$ correspondingly(see the lower hierarchies in Figure 3). By applying the procedure of calculating estimated weights for Type $0_{i}$, we obtain the estimated weights ${ }_{0_{1}} \Omega_{j}^{0}$ for Type $0_{1}$, Type $0_{2}, \ldots$, Type $0_{s}$ corresponding to the Type 1, Type 2,.., Type s hierarchy structures. Consider $T_{i}^{0}$ as being the sum of the absolute deviation between the weights ${ }_{0_{1}} \Omega_{j}^{0}$, j=1 to $\mathrm{m}_{0}$ of Type $0_{i}$ and the average of all Type $0_{i}$, $\mathrm{i}=1$ to $\mathrm{s}$, in the 0th iteration of the compromised process. $T_{i}^{0}$ and $(C I)^{0}$ are defined as follows:

$$
\begin{aligned}
& T_{i}^{0}=\sum_{j=1}^{m_{0}}\left|0_{i} \Omega_{j}^{0}-\frac{\sum_{i=1}^{S} 0_{i} \Omega_{j}^{0}}{s}\right|, \\
& (C I)^{0}=\sum_{i=1}^{S} T_{i}^{0} \quad \mathrm{i}=1 \ldots \mathrm{s}
\end{aligned}
$$

$T_{i}^{0}$ is an index to measure the degree of nearness between the weights of the Type $0_{i}$ hierarchy structure and the average of all hierarchy structures. The larger a value of the $T_{i}^{0}$ index is, the more weights of Type $0_{i}$ are deviated from the average. So the weights of Type $0_{i}$ seems more biased (inconsistent) than 
the other types. That the maximum value of $T_{i}^{0}$ is $2(1-1 / \mathrm{s})$, makes the value $2(1-1 / \mathrm{s})-T_{i}^{0}$ and its normalized index $K_{i}^{0}$ are defined as the undeviating index of Type $0_{i}$ in the 0 th iteration.

$$
K_{i}^{0}=\frac{2\left(1-\frac{1}{S}\right)-T_{i}^{0}}{\sum_{i=1}^{S}\left(2\left(1-\frac{1}{S}\right)-T_{i}^{0}\right)} \quad \mathrm{i}=1 \ldots \mathrm{s}
$$

The compromised weights of the one level hierarchy structure in the 0th iteration are calculated as follows:

$$
0^{W_{j}^{0}}=K_{1}^{0} 0_{1} \Omega_{j}^{0}+K_{2}^{0} 0_{2} \Omega_{j}^{0} \ldots \ldots+K_{S}^{0} 0_{S} \Omega_{j}^{0} \quad \mathrm{j}=1 \ldots . \mathrm{m}_{0}
$$

After the compromised weights of the one level hierarchy structure have been obtained $\left({ }_{0} W_{j}^{0}\right)$, the estimated weights ${ }_{1} \Omega_{j}^{0},{ }_{2} \Omega_{j}^{0}, \ldots,{ }_{s} \Omega_{j}^{0}$ for Type 1, Type 2,..., Type $s$ are derived. Applying the same procedure, the compromised weights of Type 1 , Type $2, \ldots$, Type $s$ in the first run are obtained by applying the following convex combination:

${ }_{i} W_{j}^{1}=K W_{j}^{0}+(1-K){ }_{i} \Omega_{j}^{0} \quad, \mathrm{i}=1 \ldots \mathrm{s}, \mathrm{j}=1 \ldots \mathrm{m}_{s}$ Continuing the stated steps in the rth
the
iteration,
weights $\Omega^{r}, \Omega^{r}, \ldots, \Omega^{r}$, the $T_{i}^{r}$ index, the normalized weights $K_{i}^{r}$ for Type $0_{i}, \mathrm{i}=1 \ldots \mathrm{s}$, and the compromised weights ${ }_{0} W_{j}^{r}$ are obtained as follows:

$$
\begin{aligned}
& T_{i}^{r}=\sum_{j=1}^{m_{0}}\left|0 \Omega_{i}{ }_{j}^{r}-\frac{\sum_{i=1}^{S} 0{ }^{2} \Omega_{j}^{r}}{S}\right| \\
& (C I)^{r}=\sum_{i=1}^{S} T_{i}^{r} \quad \mathrm{i}=1 \ldots \mathrm{s} \\
& K_{i}^{r}=\frac{2\left(1-\frac{1}{S}\right)-T_{i}^{r}}{\sum_{i=1}^{S}\left(2\left(1-\frac{1}{S}\right)-T_{i}\right)} \quad \mathrm{i}=1 \ldots \mathrm{s} \\
& 0^{W_{J}^{r}}=K_{1}^{r} \quad 0_{1} \Omega_{j}^{r}+K_{2}^{r} 0_{2} \Omega_{j}^{r} \ldots \ldots+K_{S}^{r} 0_{S} \Omega_{j}^{r} \quad \mathrm{j}=1 \ldots \mathrm{m}_{0} \\
& { }_{i} W_{J}^{r}=K_{i} W_{j}^{r-1}+(1-k)_{i} \Omega_{j}^{r-1} \quad \mathrm{i}=1 \ldots \mathrm{s}, \mathrm{j}=1 \ldots \mathrm{m}_{\mathrm{s}}
\end{aligned}
$$

In repeating the stated procedures, the compromised weights ${ }_{0_{i}} \Omega_{j}^{r} \backslash{ }_{0} W_{j}^{r} \backslash{ }_{i} W_{j}^{r}$, being a convergent series, $T_{i}^{r}$ will converge to zero and $K_{i}^{r}$ will be equal for all Type $0_{i}$ eventually. Therefore these two convergent properties can be regarded as the stopping rules of the multiple structures model of HCA. Finally, we obtain a set of consistent weights for the hierarchy structures of all types in line with Equation (1); that is; an agreeable, aggregated evaluation index for the hierarchy structures of all types will be obtained.

\section{Example}

The motivation for this work was originated in 2004 when Chung Yuan Christian University in Taiwan attempted to set up a set of performance evaluation model for its faculty. A committee was formed to choose the most suitable analytical model. Initially, the AHP was suggested, but due to a need to represent different evaluation viewpoints and subsequent hierarchy structures, it was soon discovered that extended HCA should be used. The procedure of implementing the HCA to build up the multiple structures model of AHP.is:

Step 1. Defining the lowest level criteria for evaluating the problem

Step 2. Setting up hierarchy structures according to the different evaluation viewpoints

Step 3. For each hierarchy structure input data are collected through pair-wise comparisons of interrelated criteria belonging to the same cluster and level. Next, the eigenvalue method is used to estimate the weight of criteria, and the consistency ratio is calculated to judge the rationality of the DM. Pair-wise comparisons are revised in case the ratio is too high.

Step 4. The HCA compromise process is used to obtain consistent weights for all hierarchy structures, and the consistency index is calculated to judge the rationality of the DM. Step 3 is repeated in the case when the consistency index is too high.

Step 5. The final weights are multiplied by their corresponding observed values to obtain ratings for the evaluated entity. Furthermore, an insight into the weight structure of the evaluated problem is provided both before and after compromised process

\section{Conclusion}

The contribution of this paper is to establish a multiple structures model for AHP through extension of HCA. Both AHP and HCA are weighting methods for hierarchy evaluation problems. The former has been widely studied in large-scale problems. The later is a new approach which has a different design concept and properties. The HCA approach has the advantages of providing a comprehensive framework for solving multiple hierarchy evaluation problems, providing a simpler method for calculating the weight of criteria, providing a consistency test to monitor DM judgment and delivering more hierarchy types structures to aid $\mathrm{DM}$ in understanding the performance status of the evaluated system. The distinctions between 
the two approaches are summarized in Table 1.

Table 1. The distinctions between AHP and HCA approach

\begin{tabular}{|l|c|c|}
\hline & AHP & HCA \\
\hline Consistency test & provided & provided \\
\hline Solution method & Eigenvalue method & Compromise algorithm \\
\hline Input data & $\begin{array}{c}\text { Makes a series of pair-wise comparisons } \\
\text { between each interrelated criteria }\end{array}$ & Assigns weights to criteria directly \\
\hline Evaluation reference & $\begin{array}{c}\text { based on a single structure } \\
\text { (single viewpoint) }\end{array}$ & $\begin{array}{c}\text { based on multiple structures } \\
\text { (multiple viewpoints) }\end{array}$ \\
\hline
\end{tabular}

The advantage of the multiple structures model of AHP is to avoid the problem of the selection of evaluation hierarchy structure, and to use multiple evaluation hierarchy structures to mitigate possible structure bias. Furthermore, the integration model provides a double check to test whether the DM is consistent or rational when assigning the criteria weights for the hierarchy structures. The AHP approach uses the inside information (the pair-wise comparisons of interrelated criteria within a hierarchy structure) and HCA approach utilizes outside information (comparison and compromise the criteria weight among hierarchy structures) to judge the consistency of the DM. Under these two types of index monitoring, the evaluated results should be more objective and reasonable than using either the AHP or HCA approaches separately and singly.

The multiple structures model of AHP offers more types of performance evaluation structures, and this gives the DM more insight into the problem being considered. This is more practical and helpful to the DM in understanding and auditing the performance status of a system from different angles. Further, when more structure types are available, the estimate of the evaluation problem criterion weights will have less structure bias and are more precise.

The multiple structures model of AHP is a multiple-criterion and a multiple-structure model. It can be further designed to allow a group of DMs to establish their evaluation hierarchy structures individually according to different viewpoints, and then use the AHP and HCA processes to obtain consistency weights. Implicit in the multiple structures model of AHP, the approach can be developed into a group decision methodology and can be easily applied.

The multiple structures of AHP model which combines the advantages of AHP and HCA approaches is especially suited for large complex problems where different hierarchy structures occur frequently and naturally. In a sense, due to this natural occurrence, there is no added work needed to analyze the additional structures.

The multiple structures of AHP model can be regarded as a generalized AHP model, hence the AHP model based on only one hierarchy structure can be considered a special case of the proposed method. Under these circumstances, the proposed method seems to have a natural advantage.

\section{References}

[1] E.N. Weiss and V.R. Rao(1987) "AHP Design Issues for Large scale Systems" Decision Science, Vol.18, No.1, pp.43-61.

[2] T.L. Saaty(1978)"Modeling unstructured decision hierarchies" Mathematics and Computers in Simulation, Vol.21, No.1, pp.147-157.

[3] T.L. Saaty and E. Erdener(1979)"A New Approach to Performance Measurement - The Analytical Hierarchy Process" Design method and theories, Vol.13, N0.2, PP.64-72.

[4] Y.Y. Guh, E.S.Lee \& K.M.Wang (1996) "Hierarchies Consistency Analysis for Non-transitive Problems." Computers and Mathematics with Applications, Vol.32,Vol.9, pp.67-77.

[5] Y.Y. Guh (1996)“Determining Weight by Combining Different Hierarchy StructureHierarchies Consistency Analysis” International Journal of Information and Management Science Vol.7, No.2, pp.63-80.

[6] Y.Y. Guh (1997) "Introduction to A New Weighting Method - Hierarchies Consistency Analysis" European Journal of Operational Research, Vol.102, pp.215-226. 